\title{
Perspectives, mythes et réalités en France
}

\section{Pierre-Louis Gauthier}

\section{OpenEdition}

Journals

Édition électronique

URL : http://journals.openedition.org/ries/3383

DOI : $10.4000 /$ ries.3383

ISSN : 2261-4265

\section{Éditeur}

Centre international d'études pédagogiques

\section{Édition imprimée}

Date de publication : 1 mars 1996

Pagination : 31-39

ISSN : 1254-4590

\section{Référence électronique}

Pierre-Louis Gauthier, «Perspectives, mythes et réalités en France », Revue internationale d'éducation de Sèvres [En ligne], 09 | 1996, mis en ligne le 19 août 2013, consulté le 23 mars 2021. URL : http:// journals.openedition.org/ries/3383 ; DOI : https://doi.org/10.4000/ries.3383

Ce document a été généré automatiquement le 23 mars 2021.

(c) Tous droits réservés 


\title{
Perspectives, mythes et réalités en France
}

\author{
Pierre-Louis Gauthier
}

1 La mondialisation des phénomènes sociaux, économiques et culturels, à laquelle n'échappe pas l'éducation, contraint désormais à aborder tout fait éducatif dans une perspective internationale et comparative.

2 L'introduction des langues dans l'enseignement primaire est un phénomène généralisé dans les pays de l'Union européenne récemment élargie aussi bien que dans les pays non encore représentés, de la Norvège à la Suisse et à l'Europe centrale. Les préalables sont partout les mêmes : à la mondialisation des échanges et des communications, à l'émergence de la citoyenneté européenne, s'ajoutent des enjeux de politique interne liés au développement de vastes régions souvent transfrontalières. Partout s'imposent, auprès de l'anglais comme langue de communication internationale, une ou deux langues de grande communication comme l'allemand, le français ou l'espagnol. Sur cet ensemble assez uniforme, se greffent des variantes propres aux situations nées de la proximité géographique et culturelle, en particulier dans les régions périphériques. Telle est la situation de l'allemand et du français en Alsace, Moselle et BadeWurtemberg, au Luxembourg, en Belgique germanophone, en Suisse dialectophone, dans le Val-d'Aoste, dans le Haut-Adige, du catalan de Barcelone à la Sardaigne, du basque, du suédois en Finlande, etc.

3 L'environnement linguistique de chaque pays crée donc une problématique originale qui appelle des solutions scolaires diverses, en permanente évolution.

4 Les décisions concernant l'enseignement des langues étrangères au primaire se fondent sur un primat lié aux travaux et recherches en psycholinguistique des dernières décennies : la précocité est un atout important pour l'acquisition d'une langue seconde. Par précocité ${ }^{1}$, il faut entendre le début de l'apprentissage fixé avant le secondaire. On connaît en effet le comportement de l'adolescent dont les facultés cognitives sont bien supérieures aux facultés d'expression et dont les codes de communication sont éloignés de ceux de l'adulte-professeur. La conséquence de ce déséquilibre est que les adolescents se désintéressent des situations forcément simples des cours de langues 
pour débutants basées sur des dialogues jugés «enfantins ». De même, ils trouvent rapidement artificiels et ennuyeux les exercices structuraux. L'enfant prépubertaire, lui, ne connaît pas ces inhibitions et se prête volontiers à ces "comédies" pour débutants. Sa participation stimule au contraire son goût pour les jeux de rôles. La langue étrangère est pour lui un objet ludique et de plaisir. Ajoutons que la connaissance précoce d'une langue étrangère a probablement une influence bénéfique sur la maîtrise de la langue maternelle grâce à un emploi plus conscient et comparatif. L'apprentissage précoce d'une langue étrangère a été, de longue date, l'apanage des élites sociales. C'est récemment que l'allongement de la scolarité obligatoire, réalisé partout en Europe, en a fait une réalité pour le plus grand nombre. Dès 1976, le Conseil de l'Europe a multiplié les recommandations en ce sens. Le Danemark, en rendant obligatoire l'anglais dès la première année du primaire $(7$ ans $)$ en 1958, est probablement pionnier en la matière. Les Pays-Bas ont suivi la même voie en 1985, élaborant en outre le Plan national d'action ${ }^{2}$ qui depuis 1992 propose une série de trente-quatre recommandations à tous les ordres d'enseignement, du préscolaire à l'enseignement supérieur. L'Italie a introduit les langues étrangères dans le cadre de la réforme de l'enseignement primaire (1992). La Grande-Bretagne avait assez largement développé l'enseignement du français à partir de 1963, jusqu'au coup d'arrêt, dix ans plus tard, à la suite de la publication du rapport d'évaluation Primary French in Balance. En Allemagne, certains Länder ont pris des initiatives dans les Grundschulen, avec le souci de développer les relations transfrontalières. En Irlande, l'anglais est obligatoire. Dans les cantons suisses dialectophones où se parle le schwytser Dütsch, l'enseignement de l'allemand est imposé depuis 1970.

5 Avec l'introduction de l'enseignement précoce, se pose évidemment le choix des langues à étudier. Là encore, les traditions culturelles, les impératifs politiques et économiques pèsent sur les orientations et décisions finales. En effet, peu de pays européens sont monolingues comme la France (en dépit de ses variantes régionales : alsacien, dialecte mosellan, créole, etc.). La plupart font usage officiel de langues régionales fortement implantées et/ou de langues nationales de proximité. Il s'ensuit, pour les systèmes éducatifs, un empilement de langues dont l'enseignement est confié à l'école primaire. Ainsi le Luxembourg, en introduisant un enseignement obligatoire de l'allemand dès la première année (six ans), suivi de celui du français, parallèlement à l'usage oral du luxembourgeois, a initié un modèle plurilinguistique particulièrement intéressant.

Ces ensembles linguistiques stratifiés coexistent en outre avec les langues de l'immigration qui appartiennent désormais au paysage linguistique de tous les pays européens, avec l'arabe, les dialectes arabophones, le portugais, le turc, le chinois, le coréen, le hindi, le malais, etc. Au Luxembourg, toujours, où $30 \%$ de la population est issue de l'immigration, l'étude des langues de l'immigration se superpose à l'ensemble ou prend la place de l'une ou l'autre langue.

7 Ainsi s'opèrent dans chaque pays des choix qui se rapprochent en fait de la mosaïque linguistique européenne. Certes, les partisans du «tout-anglais » voient toujours dans cette dernière langue un nouvel esperanto. Mais, dans cette perspective exclusivement utilitariste, c'est faire litière de l'extrême diversité linguistique qui est l'un des fondements culturels de l'Europe. Doit-on rappeler que dès 1969, une résolution des ministres du Conseil de l'Europe soulignait que cette diversité fait partie du patrimoine européen? Le rapport Piket surenchérissait en 1977 en dénonçant le mythe de la 
«hiérarchie des langues ». Auprès de l'anglais, choix certes incontournable, les différents systèmes éducatifs préconisent donc l'enseignement d'une palette élargie de langues. Pour ce faire, dans nombre de pays, ce sont deux, voire trois langues que l'élève doit aborder pendant le cycle élémentaire de la scolarité obligatoire.

\section{Quid pour la France?}

8 En France, très tôt, ont été initiées plusieurs tentatives d'enseignement précoce des langues vivantes. Ainsi, dans la foulée du traité franco-allemand de 1963, des expériences d'apprentissage de l'allemand, menées dès 1967, concernèrent en France plusieurs dizaines de milliers d'enfants dans le primaire et le préscolaire. L'expérience Sing und Spiele mit, mise en œuvre dans la région de Besançon, fut même adoptée comme méthode d'apprentissage dans le Valais suisse. Le choix de l'allemand, en France, était renforcé par le fait que la filière d'excellence en mathématiques et en sciences des lycées coïncidait souvent avec cette première langue. Des difficultés diverses (manque de financement, lacunes dans le suivi vertical jusqu'au collège, répartition inégale sur le territoire...) amenèrent les autorités à mettre fin à cette «prolifération d'expériences pédagogiques » en $1973^{3}$. Précisons qu'un enseignement expérimental de l'anglais avait été mis en place à la même période, dans quatre académies, suivi d'une évaluation en 1980. Force est de reconnaître qu'auprès d'un certain foisonnement des expériences nées souvent d'initiatives individuelles (inspecteurs, directeurs d'école, instituteurs, parents d'élèves...) ou collectives (municipalités dans le cadre de jumelages), peu de moyens furent dégagés et qu'aucune coordination ne tenta d'en assurer le développement national. En outre faisait également défaut, à l'époque, le matériel pédagogique adapté. La suppression des postes de langues dans les écoles normales, en arrêtant toute formation pour les instituteurs, allait porter un coup d'arrêt définitif à ces premières tentatives (voir plus loin). L'enseignement de l'allemand en primaire ne devait reprendre qu'en 1987, à la faveur d'une décision politique bilatérale qui comportait un enseignement du français chez notre "principal partenaire européen $»^{4}$. Cette nouvelle initiative, réellement structurée cette fois, définissant avec précision horaires, objectifs, public-cible (cours moyen: tranche d'âge dix/onze ans) et assortie d'un programme indicatif, ne présentait cependant aucun caractère obligatoire. Parmi les conditions d'implantation de cet "enseignement précoce de l'allemand ${ }^{5}$ figuraient notamment des conditions relatives à la proximité géographique, culturelle et scolaire d'établissements assurant le suivi en secondaire, ainsi qu'avec des établissements allemands (échanges). Des dispositions particulières s'appliquaient aux enfants d'expression dialectale de l'académie de Strasbourg et du département de la Moselle. Ce texte officiel peut être considéré comme le texte fondateur de l'enseignement précoce des langues vivantes en France (EPLV). Dans le corps du texte, on évoque également "l'initiation à l'allemand ", cette oscillation entre « initiation » et « apprentissage » laissant subsister un débat déjà ancien entre pédagogues et linguistes.

9 Il faudra attendre la rentrée scolaire de 1989 pour voir l'« expérimentation contrôlée " s'étendre sur les mêmes bases à l'anglais, l'espagnol, l'italien, le portugais, en fixant à $10 \%$ les effectifs de cours moyen qui devaient être concernés ${ }^{6}$. Le pilotage de l'ensemble était confié aux différents corps d'inspection. Il aura donc fallu une 
trentaine d'années pour en arriver là. Entre temps, la France avait cessé d'être parmi les pionniers.

10 Au fil des années, on précise et encadre un peu plus l'expérimentation. Dès la rentrée scolaire de 1991, l'objectif général à atteindre pour l'initiation d'une langue étrangère, est relevé à $25 \%$ des élèves de cours moyen ${ }^{7}$. Perdure aussi le débat à propos des différentes conceptions liées à l'apprentissage d'une langue étrangère. En 1991 toujours, on règle son compte à la notion de «sensibilisation » à une langue étrangère dont on dénonce le "flou et l'inefficacité » face à la "structuration en fonction d'objectifs terminaux " d'un véritable enseignement d'initiation ${ }^{8}$. L'enseignement à l'école primaire est soigneusement distingué de l'apprentissage systématique au collège. Toutes ces précisions et redondances tentent de remédier aux déviations et dérapages identifiés ici et là par les corps d'inspection au travers des grilles d'évaluation académiques et départementales. Ces tentatives de recadrage tentent également de pallier l'absence de formation spécifique des maîtres concernés et les faiblesses d'un système basé sur le volontariat plus que sur les compétences, le recrutement s'effectuant sur la base de critères assez flous. Apparaît, néanmoins, une première préoccupation de formation avec le souhait que soient mises en place des "formations pédagogiques", mais sans plus de précision... Quoi qu'il en soit, les ambitions officielles sont confirmées et le cap maintenu.

11 Dès la rentrée de septembre 1993, un demi-million d'élèves sont engagés dans l'entreprise avec un avantage pour le secteur privé (12\%), par rapport au public $(7,7 \%)^{9}$. Les années suivantes voient se confirmer le recentrage sur le cours moyen $2^{\mathrm{e}}$ année : $55,5 \%$ des élèves en $1994-1995^{10}$. Un nouveau seuil est franchi en septembre 1995 avec l'application du Nouveau contrat pour l'école et la mise en œuvre au début

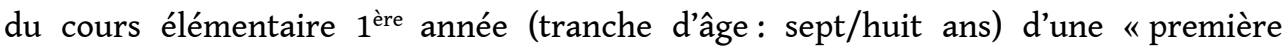
sensibilisation à une langue étrangère $»^{11}$. On revient donc sur cette notion, jadis pourfendue, dans le cadre pourtant réaffirmé de «l'enseignement d'initiation aux langues étrangères » (EILE). Le débat n'est donc pas clos! La doctrine officielle confirme la nécessaire précocité de cet enseignement et la primauté de l'oral à cet âge. Elle préconise la relation avec d'autres champs disciplinaires, l'exploitation des échanges avec des établissements étrangers, la collaboration avec des collègues étrangers. Enfin, le choix des langues s'élargit à une sixième langue : l'arabe. Cet élargissement est sans doute une disposition importante sinon la pièce essentielle du nouveau dispositif. En effet, actuellement ${ }^{12}$ l'EILE est en fait réservé à l'anglais pour $72 \%$ suivi de l'allemand (22 \%), de l'espagnol (2,6\%), de l'italien (1,8\%), de l'arabe (0,9\%). À propos du portugais $(0,3 \%)$ on a pu parler de "naufrage lusitanien ». Et que dire du russe $(0,1 \%)$ ? Le rapport de la commission des affaires culturelles du Sénat ${ }^{13}$ s'émeut de cette hégémonie de la langue anglaise à l'école primaire. Il fixe un délai de dix années pour qu'une réaction ait lieu avant d'atteindre le point de non-retour définitif de cette évolution. Le texte ministériel semble bien reprendre à son compte cette nécessaire mobilisation en faveur d'une diversification des langues et notamment des langues " modimes ${ }^{14}$.

\section{Quelle formation pour quel enseignant?}

12 On aura remarqué, chemin faisant, que le dispositif d'enseignement des langues étrangères à l'école primaire, est resté orphelin d'un vrai dispositif de formation, incluant la formation initiale des maîtres. 
13 Pourtant, à travers la doctrine confortée et précisée au fil des années, se dessine une pédagogie des langues étrangères qui non seulement se distingue de l'apprentissage systématique du secondaire, mais encore laisse se profiler un nouveau type d'enseignant.

Certes les recommandations, fréquemment réitérées, ne dépassent guère les aimables principes d'une pédagogie générale bien comprise : faire varier l'organisation de la classe, adapter les contenus aux possibilités des enfants... ${ }^{15}$. Cependant de nouvelles exigences se font jour peu à peu. Implicitement ou non, il est demandé au maître de langue, spécialiste ou généraliste, d'acquérir des notions de cognitivisme, une connaissance des théories de l'apprentissage appliquées aux jeunes enfants. Au plan des connaissances linguistiques, on souhaite non seulement un niveau mieux que moyen, mais également la connaissance des phonologies concernées. On souhaite également une bonne connaissance de la civilisation et de la culture de la langue enseignée, y compris dans les aspects de la vie quotidienne et les activités ludiques et musicales puisque c'est à la faveur de ces situations langagières que l'enseignement doit se faire à l'âge tendre. En outre, la didactique de l'enseignement des langues étrangères, particulière à l'école primaire, passe par l'intégration des autres champs disciplinaires dont le maître est également le dispensateur. On vise également l'intégration de l'enseignement des langues dans le projet global de l'établissement, ainsi que l'ouverture sur les échanges avec l'étranger.

15 Enfin, une fois de plus, l'école et l'enseignant sont placés au cœur d'un enjeu politique clairement identifié16 ${ }^{16}$ former l'enfant à cette nouvelle citoyenneté qui permettra à l'Européen de demain de participer au débat démocratique sur son continent ${ }^{17}$.

16 On voit bien comment émergent, comme toujours en pédagogie lorsqu'on introduit des procédures transversales, de nouveaux contenus, de nouveaux savoirs, un nouvel enseignant.

17 Auprès de ces ambitions, au demeurant fort légitimes dans le monde actuel, quelle est la réalité ?

18 Pour l'instant, le recrutement des enseignants passe par l'agrément des corps d'inspection, sur la base du volontariat d'une part, des compétences personnelles d'autre part assorties ou non de titres universitaires: en fait, en l'absence d'une formation systématique, à valeur contractuelle, il est malaisé d'imposer un contrôle, une exigence de niveau précis et standardisé. Contenus, méthodes, progressions, sont laissés à l'appréciation du maître volontaire. Il en va de même des intervenants que, face à la carence de l'école, recrutent les collectivités territoriales (communes, départements, régions), soucieuses de répondre à la demande des parents, leurs procédures pédagogiques et les résultats obtenus restant flous et incertains.

19 Ce pragmatisme contraste singulièrement avec l'ambition des orientations décrites plus haut. L'écart risque de se creuser plus encore si on ne complète pas l'ensemble avec un dispositif de formation assurant l'égalité des enseignants devant la nouvelle mission qui leur est dévolue et garantissant, comme le veut la loi et l'éthique de l'école publique, l'égalité des enfants devant l'éducation sur tout le territoire de la République.

20 La pierre d'achoppement du système mis en place reste donc bien et d'une manière sans cesse plus évidente au fur et à mesure que croissent les exigences, la formation des enseignants. 
21 Historiquement, les écoles normales ont assuré la formation des instituteurs en langues vivantes. Héritée de l'antique préparation des élèves-instituteurs aux épreuves du baccalauréat, cette formation était de type secondaire, c'est-à-dire ignorante des besoins des élèves du primaire. Elle a pris fin lorsque le recrutement après le baccalauréat se fut généralisé, dans les années soixante-dix. Paradoxalement, la suppression des postes qui s'ensuivit coïncida avec la montée en puissance des échanges franco-allemands et des premiers programmes européens dans les domaines scientifique et éducatif. Après la suppression des écoles normales par le ministre Jospin (1989), leur remplacement par les instituts universitaires de formation des maîtres (IUFM) semblait créer une conjoncture favorable. D'une part, les équipes des écoles normales apportaient dans la corbeille de mariage une solide expérience en matière d'échanges internationaux et un capital d'institutions étrangères partenaires (principalement des Pädagogische Hochschulen allemandes et des Polytechnics britanniques) qui pouvaient servir de moteur pour l'enseignement des langues enfin rétabli. De fait, des postes de langue furent de nouveau créés, des coordinations établies. D'autre part, le regroupement sous le même toit des personnels des premier et second degrés et en formation autorisait bien des espoirs.

Mais parmi les difficultés rencontrées par les IUFM, certaines d'entre elles allaient rapidement rendre improbable tout progrès en la matière. Tout d'abord, en délaissant les écoles d'application, lieux de toutes les expérimentations "grandeur nature » et formidable laboratoire pour l'école maternelle et l'école primaire, les IUFM se privaient, dès leur création, de cette interface particulièrement performante entre la formation, la recherche, les écoles, les maîtres, les élèves. Ensuite, en abandonnant totalement la maîtrise de la formation continue des enseignants, l'TUFM s'éloignait encore un peu plus des préoccupations et de la réflexion des acteurs du terrain pédagogique. Enfin, en ne traitant plus avec les inspections académiques qu'au travers de conventions parfois assorties de dispositions financières, les IUFM introduisaient une véritable fêlure dans le service public d'éducation.

23 La cohabitation, dans la même institution des personnels des premier et second degrés, n'a pas produit non plus cette commune culture enseignante tant espérée. L'échec du tronc commun est bien connu. On avait pourtant, en matière de langues, un champ nouveau à initier qui eût permis de fédérer les différents personnels : l'étude comparée des systèmes éducatifs européens et l'acquisition d'une véritable langue de spécialité. Mais auprès des difficultés rencontrées, il apparut que l'international n'était pas une priorité pour les directeurs d'IUFM, pas plus que l'enseignement des langues au primaire.

24 Les professeurs d'IUFM eux-mêmes, pour la plupart issus du second degré, restent avant tout des spécialistes d'une discipline, et ne sont pas sensibilisés, ni formés à une problématique transversale du métier d'enseignant comme cela se doit dans le primaire avec de jeunes enfants. L'absence d'épreuve obligatoire de langue au concours de recrutement achève de minorer aux yeux de tous cet enseignement. La place de l'EILE est donc très réduite dans le nouveau dispositif de formation initiale. Limitée à une option en première année, la langue étrangère pour le premier degré ne concerne qu'un nombre réduit d'étudiants, généralement déjà formés par l'université. L'enseignement obligatoire (vingt heures au total) tente de se mettre en place en deuxième année, malgré un manque évident de formateurs qualifiés. Le choix des langues, lui-même, se restreint aux deux ou trois langues traditionnelles du second 
degré. On comprend que la circulaire du 11 mai 1995 ne cite, parmi les moyens de formation à mettre en œuvre, qu'une seule fois les IUFM, et encore est-ce dans la perspective d'une «modification des formations ». En matière de formation continue, ce sont les corps d'inspection qui mettent en œuvre les plans de formation académiques et départementaux. Dans ces plans, la formation est souvent réduite à l'utilisation des matériels et à la «lecture" des cassettes vidéo produites par le ministère et mises à la disposition des écoles. L'enseignant se trouve dès lors cantonné dans un rôle de médiateur, peu propice à une réflexion fondamentale sur cette nouvelle mission. Quant au perfectionnement linguistique, parmi les stages européens proposés, le premier degré ne parvient pas à se faire une place en rapport avec son importance réelle dans le système éducatif.

On est encore loin du dispositif de formation pour tous qui permettrait la généralisation de l'EILE à la totalité de l'école primaire, avec de réelles garanties de qualité et d'égalité.

Indéniablement la mise en place de l'enseignement d'initiation des langues étrangères à l'école primaire constitue l'innovation la plus remarquable depuis la rénovation des enseignements de base et les nouveaux programmes. Comme toute innovation d'importance, cette nouvelle mission pour l'école conforte des procédures didactiques déjà en place: la réflexion sur la langue maternelle, la pratique systématique de l'expression orale, les échanges avec l'étranger, l'intégration des activités à un projet, etc. En outre, l'enseignement des langues fait naître chez les enseignants un sentiment d'appartenance à une communauté plus vaste en créant une dynamique d'ouverture sur d'autres cultures, d'autres systèmes éducatifs.

Tout le monde s'accorde sur les intentions des récents textes officiels : précocité de l'enseignement, choix plus diversifié de langues, continuité de l'apprentissage.

Pourtant l'épreuve de la réalité a révélé contradictions et difficultés :

- contradiction entre une visée humaniste et citoyenne menant au plurilinguisme et l'apprentissage d'une langue unique, l'anglais, obéissant surtout à des buts utilitaristes ;

- contradiction entre l'accord sur les principes de l'enseignement précoce et le peu de reconnaissance, voire l'absence de suivi, accordé à cet enseignement par le secondaire ${ }^{18}$;

- difficultés d'obtenir et de maintenir un niveau satisfaisant tout au long de la scolarité, pour des enseignants insuffisamment formés et de surcroît mis en concurrence avec d'autres catégories d'intervenants ${ }^{19}$.

29 Une grande partie de ces problèmes trouvera une solution dans la mise sur pied d'une formation initiale obligatoire en langues, accompagnée d'une certification dûment identifiée.

30 En effet, seule une formation pédagogique spécifique pourra procurer aux futurs maîtres les compétences linguistiques adaptées au jeune enfant. La formation peut être le creuset de la diversification linguistique en réaction contre la fatalité ambiante du tout-anglais, en valorisant ou en proposant une langue seconde de communication, voire une langue « modime » lorsque l'opportunité se présente.

31 Enfin, seule la formation peut apporter la dimension culturelle à l'apprentissage des langues, en s'articulant sur une culture des échanges, et ainsi élever cet enseignement nouveau à la hauteur des enjeux sans cesse rappelés d'une école ouverte sur la compréhension des autres et la citoyenneté européenne. 


\section{NOTES}

1. Certains spécialistes préfèrent voir se substituer au terme "précoce » celui de "présecondaire».

2. Nationaal Actieprogramma moderne Vreemde Talen, Dutch National Action Programme on Foreign Languages, Recommandations and Policy Reactions, Enschede, 1992.

3. Circulaire ministérielle $\mathrm{n}^{\circ} 73-228 \mathrm{du} 11$ mai 1973.

4. La note de service $n^{\circ}$ 87-035 préconise de « développer chez les enfants, à ce niveau, un intérêt pour l'apprentissage de la langue de l'un de nos principaux partenaires ».

5. Note de service $n^{\circ} 87-035$ du 15 janvier 1987, Enseignement précoce de l'allemand.

6. Circulaire $\mathrm{n}^{\circ}$ 89-065 du 6 mars 1989, Expérimentation contrôlée de l'enseignement d'une langue vivante à l'école élémentaire (écoles publiques et écoles privées sous contrat).

7. Circulaire n 90-070 du 26 mars 1990, Expérimentation contrôlée d'une langue vivante à l'école élémentaire (écoles publiques et écoles privées sous contrat).

8. Circulaire $n^{\circ}$ 91-246 du 6 septembre 1991, Expérimentation contrôlée de l'enseignement d'une langue vivante étrangère à l'école élémentaire (écoles publiques et écoles privées sous contrat) : orientations pédagogiques.

9. Note d'information, ministère de l'Éducation nationale, direction de l'Évaluation et de la Prospective, décembre 1994.

10. Note d'information, ministère de l'Éducation nationale, de l'Enseignement supérieur et de la Recherche, direction de l'Évaluation et de la Prospective, novembre 1995.

11. Circulaire n 95-103 du 3 mai 1995, Enseignement des langues vivantes.

12. Voir note 10.

13. Sénat, commission des Affaires culturelles, Rapport sur l'enseignement des langues vivantes en France, 20 décembre 1995.

14. Moins diffusées et moins enseignées.

15. Voir note 10.

16. Voir note 10.

17. Françoise Buffet, Jacques-André Tschoumy, Choc démocratique et formation des enseignants en Europe, Presses universitaires de Lyon, 1995.

18. On peut également s'interroger sur l'avenir de l'opération cours élémentaire 1re année (septembre 1995) dans les classes suivantes ainsi que son « raccordement » avec l'enseignement actuellement dispensé en cours moyen.

19. Dans la seule académie de Versailles, les personnels enseignant l'EILE (anglais) se répartissaient comme suit: instituteurs et professeurs d'école $125(22,2 \%)$; professeurs du secondaire 163 (29\%); intervenants extérieurs 274 (48,7\%).

\section{RÉSUMÉS}

Une présentation des différentes initiatives françaises conduit l'auteur à s'interroger sur les conditions de réussite d'un apprentissage des langues étrangères à l'école élémentaire et sur la mise en place d'un dispositif performant de formation initiale des enseignants dans les IUFM. 
INDEX

Index géographique : France

Mots-clés : école primaire, enseignement des langues, langues étrangères, enseignement précoce des langues vivantes

\section{AUTEUR}

\section{PIERRE-LOUIS GAUTHIER}

Inspecteur pédagogique régional, Inspecteur d'académie, France. 